(2) Open Access Full Text Article

ORIGINAL RESEARCH

\title{
Lentinan Inhibits AGE-Induced Inflammation and the Expression of Matrix-Degrading Enzymes in Human Chondrocytes
}

This article was published in the following Dove Press journal:

Drug Design, Development and Therapy

\section{Zhaozhen Zhang \\ Zhuqing Zha \\ Zhiwei Zhao \\ Wenjing Liu \\ Wuyin Li}

Department of Bone Surgery, Luoyang Orthopedic Hospital of Henan Province (Henan Provincial Orthopedic Hospital), Zhengzhou City, Henan Province 450000, People's Republic of China
Correspondence: Wuyin $\mathrm{Li}$

Department of Bone Surgery, Luoyang Orthopedic Hospital of Henan Province (Henan Provincial Orthopedic Hospital), No. 100, Yongping Road, Zhengzhou City, Henan Province 450000, People's

Republic of China

Tel +86-37I-88820000

Email wuyinl128@I63.com
Background: Chondrocyte-mediated inflammation is an important pathological component of osteoarthritis (OA) development. There are currently no therapies that completely reverse the development of OA. Lentinan, a type of polysaccharide derived from Lentinus edodes, has been demonstrated to possess significant anti-viral, anti-cancer, and anti-inflammatory effects, and has been recently used in the treatment of several inflammatory diseases. However, little research has focused on the pharmacological effect of lentinan in human OA. Materials and Methods: We evaluated the anti-inflammatory and anti-ROS effects of lentinan in SW1353 chondrocytes treated with AGEs using real-time polymerase chain reaction (PCR), enzyme-linked immunosorbent assay (ELISA), and the nitro oxide-specific stain DAF-FM DA. The regulatory effects of lentinan on NF- $\mathrm{BB}$ and MAPK p38 signaling were investigated via promoter assay and Western blot analysis.

Results: We found that lentinan inhibits the production of pro-inflammatory cytokines, including IL- $1 \beta$, TNF- $\alpha$, IL- 8 and the secretion of $\mathrm{PGE}_{2}$ and NO, by reducing the expression of COX-2 and iNOS in AGE-challenged chondrocytes. Lentinan also reduces AGE-induced increased expression of matrix metalloproteinases-1, -3, and -13 (MMP-1, MMP-3, MMP-13). Furthermore, lentinan has a similar effect on a disintegrin and metalloproteinase with thrombospondin motifs-4 and -5 (ADAMTS-4, ADAMTS-5). Mechanistically, lentinan reduces the activation of NF- $\mathrm{kB}$.

Conclusion: Our findings indicate that lentinan shows a protective effect against AGEinduced inflammatory response in chondrocytes. These findings suggest that lentinan is a promising agent for the treatment of OA that could be used as a dietary supplement for patients with OA.

Keywords: osteoarthritis, AGEs, lentinan, NF- $\kappa B$, chondrocytes

\section{Introduction}

Osteoarthritis (OA) is a painful degenerative joint disease afflicting millions of people around the world. OA is characterized by inflammation, pain, swelling, physical disability, and changes in cartilage tissues throughout the body, including in the hips and knees. Previous studies have demonstrated that risk factors such as aging, ${ }^{1}$ joint injury, ${ }^{2}$ and obesity ${ }^{3}$ play a vital role in the initiation and development of OA. However, there are no previous therapies that can completely impede the initiation and development of this disease. Advanced glycation end products (AGEs) result from the glycated endproducts of the maillard reaction (MR), which is a non-enzymatic reaction between sugars and proteins. ${ }^{4}$ AGEs accumulate in cells and tissues through the innate process 
of non-enzymatic glycation as well as dietary intake. Research has demonstrated that the accumulation of AGEs is an age-related effect that occurs through the binding of AGEs to the receptor for AGEs (RAGE) in human chondrocytes. ${ }^{5}$ The excessive formation of AGEs in chondrocytes plays a major role in the development of OA. It is well established that AGE stimulation increases the secretion of pro-inflammatory cytokines, such as IL-1 $\beta$, TNF- $\alpha$, and IL-8, and upregulates the production of nitric oxide (NO) and prostaglandin $\mathrm{E} 2\left(\mathrm{PGE}_{2}\right)$ by modulating the expression of inducible nitric oxide synthase (iNOS) and cyclooxygenase2 (COX-2) ${ }^{6-9}$ In addition, AGEs promote the activation of p38 as well as the phosphorylation and degradation of I $\kappa \mathrm{B} \alpha$, which leads to the activation of NF- $\mathrm{kB}$ signaling pathways, thereby inducing a cascade of pro-inflammatory cytokines and chemokines. ${ }^{10}$ Furthermore, AGEs enhance the expression of matrix metalloproteinases (MMPs) and a disintegrin and metalloproteinase with thrombospondin motifs (ADAMTS) ${ }^{11}$ These hydrolytic proteinases have a strong effect on the degradation of the cartilage extracellular matrix (ECM), which is mainly constituted by type II collagen and aggrecan. Degradation of the ECM is a primary pathological characteristic of OA. The exploration of a novel agent with fewer side effects has become an important goal for the treatment of OA.

Natural molecules from food sources have been sought to treat OA. Chondroitin sulfate (CS) is an important proteoglycan that acts as a major component of the extracellular matrix in joints. CS isolated from animal cartilage has been shown to have potential treatment efficacy. Pharmacological studies have revealed its capacity to promote the synthesis of cartilage matrix while inhibiting the synthesis of proteolytic enzymes that degrade the cartilage matrix. Purified CS possesses anti-inflammatory effects in primary cultured chondrocytes. ${ }^{12}$ Oral supplementation of CS has been recommended by the European Society for Clinical and Economic Aspects of Osteoporosis and Osteoarthritis and Musculoskeletal Diseases (ESCEO). ${ }^{13}$ The biotechnological production of CS has been explored in the cultivation of Escherichia coli strains. ${ }^{14}$ Hyaluronic acid is another main component of the cartilage ECM. Hyaluronic acid plays a central role in the maintenance of synovial fluid viscosity. Hyaluronic acid gel treatment reduces the production of key inflammatory cytokines including TNF- $\alpha$, IL- 6 , and IL-8 in cultured human chondrocytes by suppressing the NF- $\mathrm{KB}$ pathway. ${ }^{12,15}$ Pharmaceutical purified hyaluronic acid products show similar anti-inflammatory effects by countering the expression of TNF- $\alpha$ and IL- 6 in human primary chondrocytes. ${ }^{16}$ Dietary mushrooms are well known for their health benefits. Lentinan, a $\beta$-1,3-glucan, is a type of polysaccharide derived from Lentinus edodes. ${ }^{17}$ Previous research has demonstrated that lentinan exerts important antiviral, anti-cancer, and anti-inflammatory effects. ${ }^{18-21}$ As a biological response modifier (BRM), lentinan actualizes its effects by restoring and enhancing immune system responsiveness to stimulation and other physiologically active factors. These functions indicate that lentinan may have a protective effect on chondrocytes in OA. Although a large number of studies have reported the protective effects of lentinan in various diseases, few of them revealed whether lentinan possesses a beneficial effect on the progression and development of OA. In the present study, we investigated the pharmacological function of lentinan in AGE-challenged human chondrocytes to determine whether lentinan has a protective effect against the inflammatory response in human chondrocytes.

\section{Materials and Methods Cell Culture and Treatment}

Lentinan was purchased from Kaifeng Pharmaceutical Co. (Henan, China) ${ }^{22}$ as powder in a penicillin bottle. Lentinan was dissolved in PBS right before use. The chondrosarcoma SW1353 cells used in all experiments were purchased from ATCC. The cells were cultured in Leibovitz's L-15m medium (ATCC) containing 10\% fetal bovine serum. For the AGE treatment experiments, SW1353 cells were seeded into 6-well plates, grown to full confluence, and then exposed to $100 \mu \mathrm{g} / \mathrm{mL}$ AGEs in the presence or absence of 250 and $500 \mu \mathrm{M}$ lentinan ${ }^{23,24}$ for 24 hours. To measure the levels of phosphorylated $\mathrm{p} 38$ and $\mathrm{I} \kappa \mathrm{B} \alpha$, cells underwent the same treatment for 2 hours.

\section{Real-Time Polymerase Chain Reaction (PCR)}

RNA was extracted from 2-4 million SW1353 cells using a commercial RNA MiniPrep Purification Kit (Qiagen). Then, $1 \mu \mathrm{g}$ RNA was used to synthesize cDNA using an RT-PCR One-step Kit (Bio-Rad, USA). Real-time PCR was performed using the SYBR-based real-time PCR method to measure the mRNA transcripts of the target genes and GAPDH (as a housekeeping control) using the Bio-Rad platform. The expression of each of the target genes was normalized to GAPDH and calculated using the $2^{-\Delta \Delta \mathrm{CT}}$ method. 


\section{Enzyme-Linked Immunosorbent Assay (ELISA)}

Roughly $1 \times 10^{4}$ SW1353 cells were used for ELISA. To measure the protein secretions of the target genes, the supernatants were collected from the culture medium by centrifugation at $1000 \mathrm{rpm}$ for $10 \mathrm{~min}$. Commercial ELISA kits for TNF- $\alpha$, IL-1 $\beta$, IL-8, MMP-1, MMP-3, MMP-13, ADAMTS-4, and ADAMTS-5 were purchased from R\&D Systems and used in accordance with the manufacturer's instructions. The final values of the reaction results were extrapolated from the standard curve using a variable slope. The resulting data are presented as fold-changes using the non-treated group as a baseline.

\section{Western Blot Analysis}

After the indicated treatment, 4-6 million cells were lysed using RIPA buffer supplemented with protease inhibitor cocktail. Then, a total of $20 \mu \mathrm{g}$ cell lysates was loaded onto 4-20\% precasted polyacrylamide gel electrophoresis (PAGE) gel to separate the proteins according to size. The separated protein mix was then transferred onto polyvinylidene fluoride (PVDF) membranes and the corresponding protein levels were detected using their specific antibodies. The membranes were incubated overnight with primary antibodies at $4{ }^{\circ} \mathrm{C}$, and then washed and incubated with HRP-conjugated secondary antibodies for $1 \mathrm{~h}$. HRP substrate was added to detect the resulting protein signals which were visualized using Image $\mathbf{J}$ software.

\section{4-Amino-5-Methylamino-2',7'- Difluorofluorescein Diacetate (DAF-FM DA) Staining}

The intracellular production of NO was measured by performing a cellular staining experiment using the cellpermeable fluorescent probe DAF-FM DA (Thermo-Fisher Scientific, USA). Briefly, 10,000 cells were grown to full confluence on a 96-well plate, and then incubated with $5 \mu \mathrm{M}$ DAF-FM DA probe for $10 \mathrm{~min}$. The resulting fluorescent signals were visualized using a fluorescence microscope, with excitation at $495 \mathrm{~nm}$ and emission detection at $515 \mathrm{~nm}$.

\section{Luciferase Activity of NF- $\kappa B$}

The NF- $\mathrm{BB}$ bind sites containing firefly luciferase vector were used to evaluate the transcriptional activity of NF- $\mathrm{kB}$. Briefly, the cells were co-transfected with NF- $\mathrm{KB}$ promoter and a renilla luciferase promoter using Lipofectamine 3000 reagent from Invitrogen (cat \# 11668027) in accordance with the manufacturer's instructions. After the indicated treatment, the total cell lysates were collected and the dual luciferase activity of renilla and firefly luciferase was measured. The relative luciferase activity was calculated by normalizing the firefly luciferase activity to that of renilla luciferase.

\section{Statistical Analysis}

All experimental data are presented as means \pm S.E.M. in this study The statistical significance of differences was measured using one-way analysis of variance (ANOVA) followed by Bonferroni's post hoc test. A value of $\mathrm{P}<0.05$ was considered statistically significant.

\section{Results}

Measuring the Production of Pro-Inflammatory Cytokines

Proinflammatory TNF- $\alpha$, IL- 8 , and IL- $1 \beta$ are known to play a key role in the development of OA. Therefore, we tested the effects of lentinan on the production of these cytokines in AGE-induced chondrocytes. Firstly, we investigated the expression of TNF- $\alpha$, as shown in Figure 1A. The mRNA level of TNF- $\alpha$ increased to 3.9-fold with AGE treatment alone and the secretion of TNF- $\alpha$ was upregulated to 792.3 from $123.5 \mathrm{pg} / \mathrm{mL}$ at baseline (Figure 1D). However, in the presence of the two doses of lentinan, AGE stimulation increased the mRNA level of TNF- $\alpha$ to 2.8- and 2.2-fold and the secretion of TNF- $\alpha$ to 568.2 and $432.5 \mathrm{pg} / \mathrm{mL}$ (Figure 1D), respectively. Secondly, we turned to the mRNA level and secretion of IL-8. When the two doses of lentinan were added, AGEs increased the mRNA level of IL-8 to 3.4- and 2.5-fold, compared with 4.6-fold upon AGE stimulation alone (Figure 1B). Furthermore, AGEs alone increased the secretion of IL- 8 to $1544.3 \mathrm{pg} / \mathrm{mL}$, but its secretion was decreased to 1192.6 and $878.6 \mathrm{pg} / \mathrm{mL}$ when exposed to the two doses of lentinan (Figure 1E). Similarly, the mRNA level of IL-1 $\beta$ was significantly suppressed to 2.6- and 1.9-fold by the two doses of lentinan from 3.6-fold with AGE treatment alone (Figure 1C), the secretion of IL$1 \beta$ was reduced from 1099.5 to $781.5 \mathrm{pg} / \mathrm{mL}$ and $623.6 \mathrm{pg} /$ $\mathrm{mL}$ by lentinan, respectively (Figure $1 \mathrm{~F}$ ).

\section{The Expression of Matrix Metalloproteinases}

To determine the impact of lentinan on the expression of MMPs, which play a major role in the degradation of type II collagen, the mRNA and protein levels of MMP-1, MMP-3, and MMP-13 were measured. As the results in Figure 2A 
A

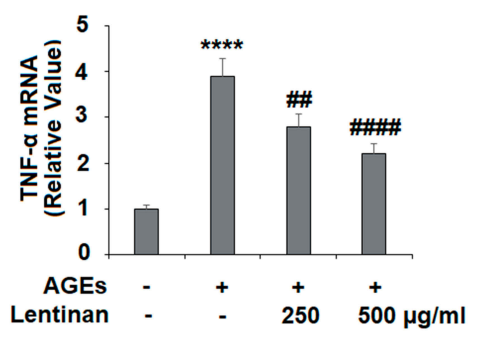

D

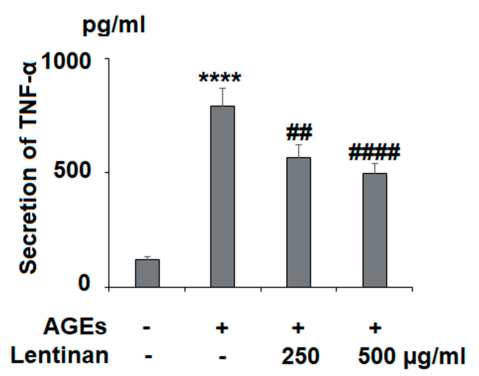

B

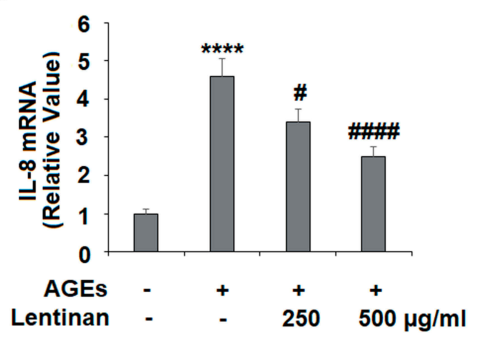

E

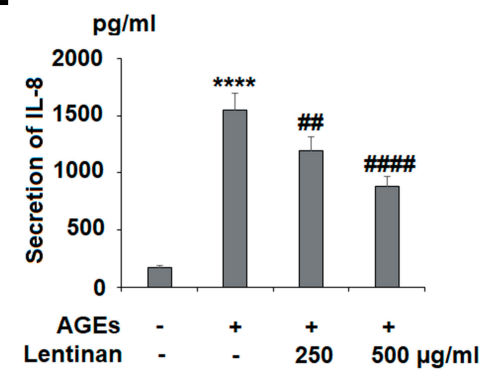

C

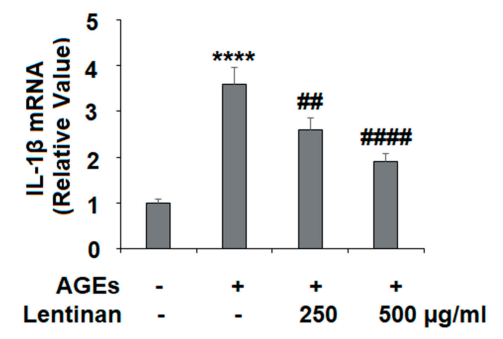

F

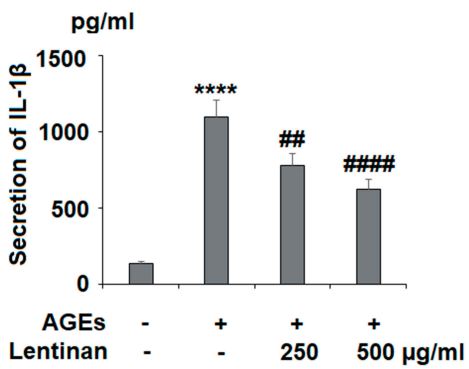

Figure I Lentinan reduced AGE-induced expression of TNF- $\alpha$, IL-8, and IL-I $\beta$ in human SWI353 chondrocytes. Cells were stimulated with I00 $\mu g / m L$ AGEs with or without lentinan $(250$ and $500 \mu \mathrm{g} / \mathrm{mL})$ for $24 \mathrm{~h}$. (A) mRNA of TNF- $\alpha$; (B) mRNA of IL-8; (C) mRNA of IL-I $\beta$; (D) Secretion of TNF- $\alpha$ as measured by ELISA; (E) Secretion of IL-8 as measured by ELISA; (F) Secretion of IL-I $\beta$ as measured by ELISA (*****P<0.000I vs vehicle group; \#, \#, \#\#, P<0.05, $0.01,0.000$ I vs AGEs treatment group, $n=4-5)$.

show, the mRNA levels of MMP-1, MMP-3, and MMP-13 significantly increased to 3.7-, 3.3-, and 4.7-fold by AGE treatment alone, respectively. However, $250 \mu \mathrm{g} / \mathrm{mL}$ lentinan reduced the mRNA levels of these three factors to 2.6-, 2.4-, and 3.2-fold, respectively. Furthermore, $500 \mu \mathrm{g} / \mathrm{mL}$ lentinan significantly reduced them to 1.5-, 1.6-, and 1.9-fold. Congruously, as shown in Figure 2B, the results of ELISA analysis show that AGE treatment increased the protein levels of MMP-1, MMP-3, and MMP-13 from 125.7, 93.7, and $143.6 \mathrm{pg} / \mathrm{mL}$ to $493.4,552.3$, and $666.9 \mathrm{pg} / \mathrm{mL}$. Meanwhile, the two doses of lentinan reduced the protein level of MMP-1 to 325.6 and $228.7 \mathrm{pg} / \mathrm{mL}$, MMP-3 to 381.2 , $299.5 \mathrm{pg} / \mathrm{mL}$, and MMP-13 to $481.2,333.5 \mathrm{pg} / \mathrm{mL}$, respectively. Taken together, these findings imply that lentinan has a strong inhibitory effect on the expression of MMPs.

\section{Expression Changes of ADAMTS-4 and ADAMTS-5}

As ADAMTS plays a major role in aggrecan degeneration, we investigated the effect of lentinan on the expression of ADAMTS-4 and ADAMTS-5. The results in Figure 3A show that the mRNA levels of ADAMTS-4 and
A

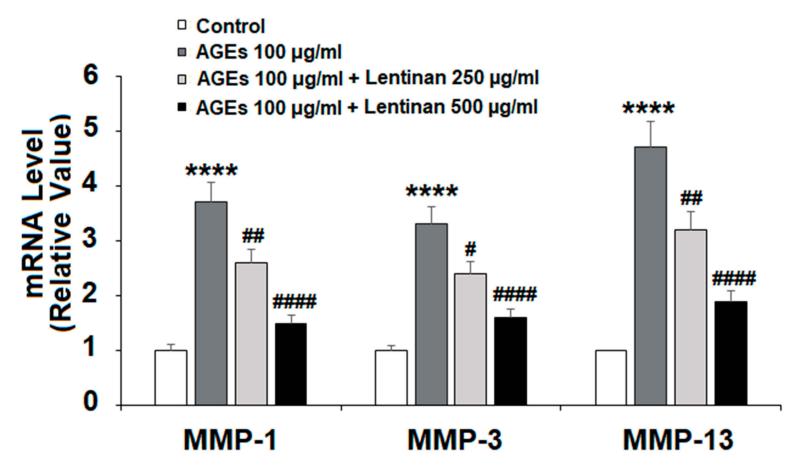

B

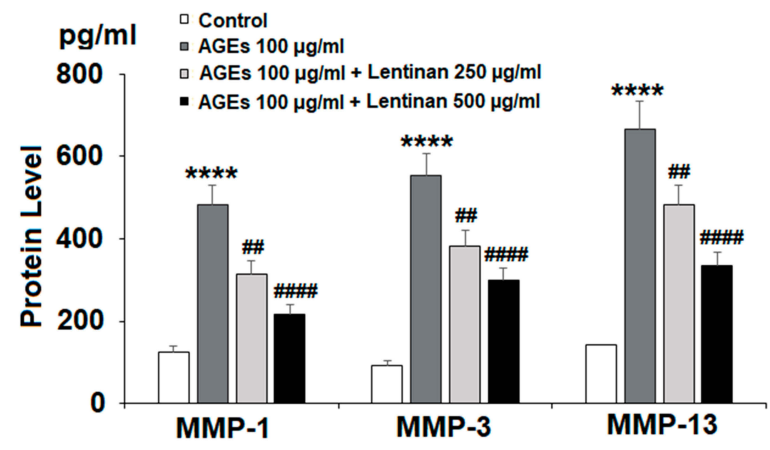

Figure 2 Lentinan suppressed AGE-induced expression of MMP-I, MMP-3, and MMP-I3 in human SWI 353 chondrocytes. Cells were stimulated with I00 $\mu$ / $/ \mathrm{mL}$ AGEs with or without lentinan $(250$ and $500 \mu \mathrm{g} / \mathrm{mL}$ ) for $24 \mathrm{~h}$. (A) mRNA levels of MMP-I, MMP-3, and MMP-I3; (B) Protein levels of MMP-I, MMP-3, and MMP-I3 as measured by ELISA (****, P<0.000I vs vehicle group; \#, \#, \#\#, P<0.05, $0.01,0.0001$ vs AGEs treatment group, $n=3-4$ ). 
ADAMTS-5 were significantly enhanced to 3.7-and 3.4-fold by exposure to AGEs alone, while $250 \mu \mathrm{g} / \mathrm{mL}$ lentinan showed an inhibitive effect on these two enzymes, reducing their mRNA expression to 2.6- and 2.2-fold, and $500 \mu \mathrm{g} / \mathrm{mL}$ lentinan further reduced them to $1.8-$ and 1.6-fold. Congruously, AGE treatment enhanced the protein levels of ADAMTS-4 and ADAMTS-5 to 582.4 and $668.9 \mathrm{pg} / \mathrm{mL}$, while $250 \mu \mathrm{g} / \mathrm{mL}$ lentinan was able to decrease them to 391.3 and $492.1 \mathrm{pg} / \mathrm{mL}$, respectively. In addition, $500 \mu \mathrm{g} / \mathrm{mL}$ lentinan further decreased the protein levels of ADAMTS-4 and ADAMTS-5 to 278.5 and $335.6 \mathrm{pg} / \mathrm{mL}$, as shown in Figure $3 \mathrm{~B}$. Our data reveal that lentinan suppresses the expression of ADAMTS-4 and ADAMTS-5 at both the mRNA and protein levels.
A

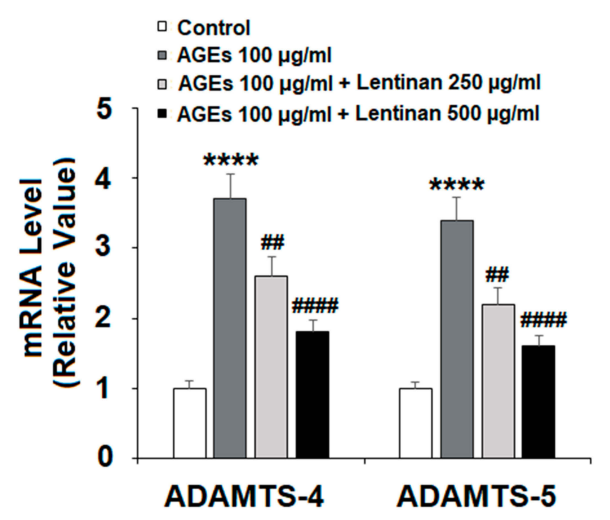

B

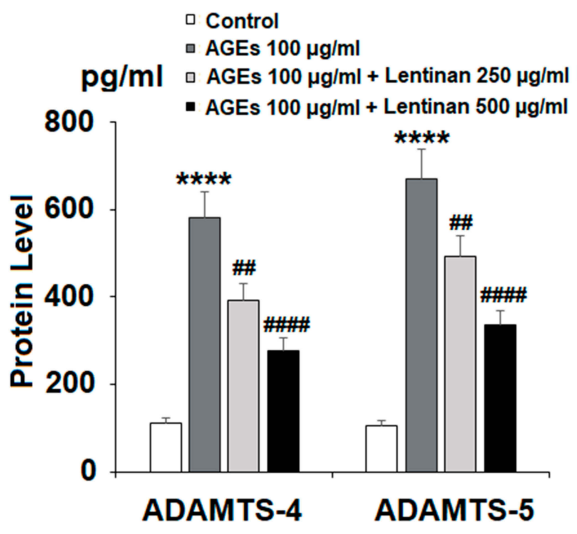

Figure 3 Lentinan inhibited AGE-induced expression of ADAMTS-4 and ADAMTS- 5 in human SWI 353 chondrocytes. Cells were stimulated with $100 \mu \mathrm{g} / \mathrm{mL}$ AGEs with or without lentinan $(250$ and $500 \mu \mathrm{g} / \mathrm{mL}$ ) for $24 \mathrm{~h}$. (A) mRNA of ADAMTS-4 and ADAMTS-5; (B) Protein of ADAMTS-4 and ADAMTS-5 as measured by ELISA (****, P<0.000I vs vehicle group; \#, \#\#, $\mathrm{P}<0.01,0.0001$ vs $A$ GEs treatment group, $n=3-4)$.

A

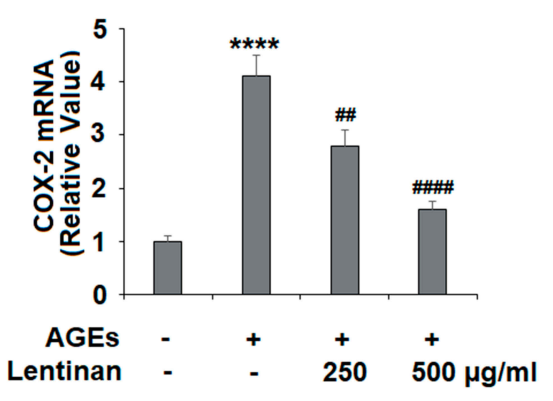

B

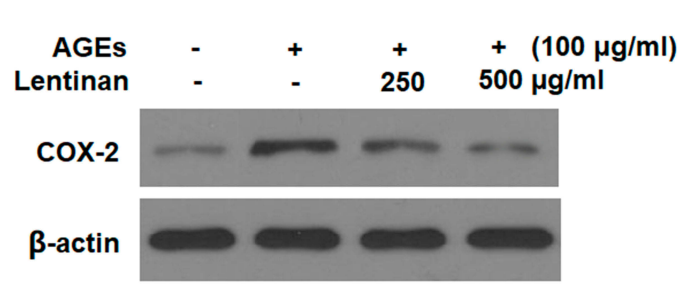

C

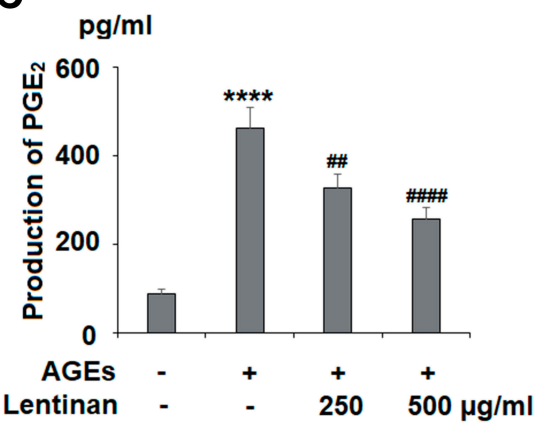

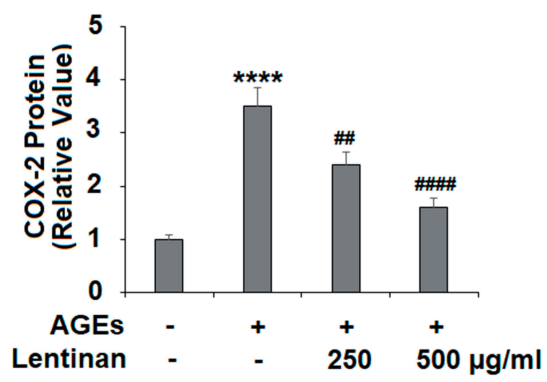

Figure 4 Lentinan prevented AGE-induced COX-2 expression and PGE 2 generation. Cells were stimulated with $100 \mu g / \mathrm{mL}$ AGEs with or without lentinan (250 and $500 \mu \mathrm{g} /$ $\mathrm{mL}$ ) for 24 h. (A) mRNA of COX-2; (B) Protein of COX-2 as measured by Western blot analysis; (C) Production of PGE 2 (****, P<0.000I vs vehicle group; \#\#, \#\#\#, $P<0.01,0.000$ I vs $A G E s$ treatment group, $n=4-5$ ). 


\section{Expression Changes of COX-2 and $\mathrm{PGE}_{2}$}

The results in Figure 4A shows that lentinan reduced the mRNA level of COX-2 to 2.8- and 1.6-fold, compared with 4.1-fold via exposure to AGEs alone, in a concentrationdependent manner. In addition, the results of Western blot analysis in Figure 4B reveal that the two doses of lentinan significantly decreased the protein levels of COX-2 to 2.4and 1.6-fold, compared to 3.5-fold with AGE treatment alone. For the production of $\mathrm{PGE}_{2}$, the baseline of the protein level of $\mathrm{PGE}_{2}$ was $88.8 \mathrm{pg} / \mathrm{mL}$, and the addition of AGEs enhanced it to $462.3 \mathrm{pg} / \mathrm{mL}$. However, two doses of lentinan were able to reduce the protein level of $\mathrm{PGE}_{2}$ to 325.8 and $256.7 \mathrm{pg} / \mathrm{mL}$, respectively (Figure 4C).

\section{Expression of iNOS and Production of NO}

In order to determine the effect of lentinan on the expression of iNOS and the generation of NO, Western blot analysis and DAF-FM staining approaches were employed. The results in Figure 5A show that stimulation with AGEs upregulated the mRNA level of iNOS to 3.1-fold from baseline, while $250 \mu \mathrm{g} / \mathrm{mL}$ lentinan reduced it to 2.7 -fold, and $500 \mu \mathrm{g} /$ $\mathrm{mL}$ lentinan further reduced the mRNA level of iNOS to 2.1-fold. As for the protein level of iNOS, the data in Figure $5 \mathrm{~B}$ reveal that the two doses of lentinan decreased the iNOS protein level to 2.1- and 1.3-fold, respectively, as compared to the exposure to AGEs alone. The results of DAF-FM staining in Figure 5C show that the two doses of lentinan significantly downregulated the protein level of NO to 2.4and 1.6-fold, respectively, as opposed to the increase to 3.4-fold induced by AGE treatment alone.

\section{Activation of P38}

In order to test whether lentinan affects the activation of the p38/MAPK pathway, the phosphorylation of $\mathrm{p} 38$ (p-p38) was tested. As shown in Figure 6, the level of p-p38 was significantly increased to 3.8-fold upon exposure to AGEs alone, while the two doses of lentinan reduced the level of p-p38 to 2.6-and 1.5-fold, respectively. These results reveal that lentinan has a potent inhibitory effect on the activation of $\mathrm{p} 38$.

\section{Phosphorylation and Degradation of $I \kappa B \alpha$}

It is well documented that the phosphorylation and degradation of $I \kappa B \alpha$ is a trigger for the activation of the NF- $\kappa B$ signaling pathway. Our data in Figure 7 show that AGEs increased the phosphorylated level of $\mathrm{I} \kappa \mathrm{B} \alpha(\mathrm{p}-\mathrm{I} \kappa \mathrm{B} \alpha)$ to 3.2-fold, while the two doses of lentinan reduced the level of $\mathrm{p}-\mathrm{I} \kappa \mathrm{B} \alpha$ to 2.1- and 1.2-fold, respectively. On the contrary, AGE treatment suppressed the total level of $\mathrm{I} \kappa \mathrm{B} \alpha$ to $34 \%$ of that of baseline. However, $250 \mu \mathrm{g} / \mathrm{mL}$ lentinan significantly increased the total level of $\mathrm{I} \kappa \mathrm{B} \alpha$ to $69 \%$ of that of base line, while $500 \mu \mathrm{g} / \mathrm{mL}$ lentinan recovered $98 \%$ I $\kappa \mathrm{B} \alpha$ level.

\section{The Effects of Lentinan on AGE-Induced NF- $\kappa B$ Activation}

As the phosphorylation and degradation of $\mathrm{I} \kappa \mathrm{B} \alpha$ is a hallmark of NF- $\kappa \mathrm{B}$ activity, we measured the nuclear translocation and luciferase activity of NF- $\mathrm{B}$ promoter. The results in Figure $8 \mathrm{~A}$ reveal that the two doses of lentinan reduced the nuclear translocation of $\mathrm{NF}-\kappa \mathrm{B}$ to 2.7- and 1.7-fold, respectively, as compared with 3.8-fold upon exposure to AGEs alone. The luciferase activity of
A

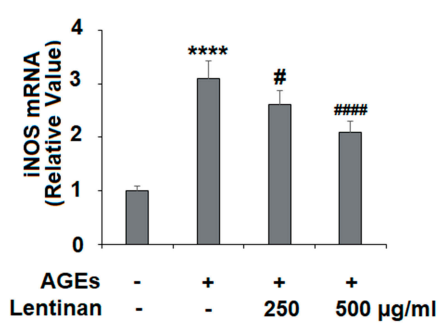

B

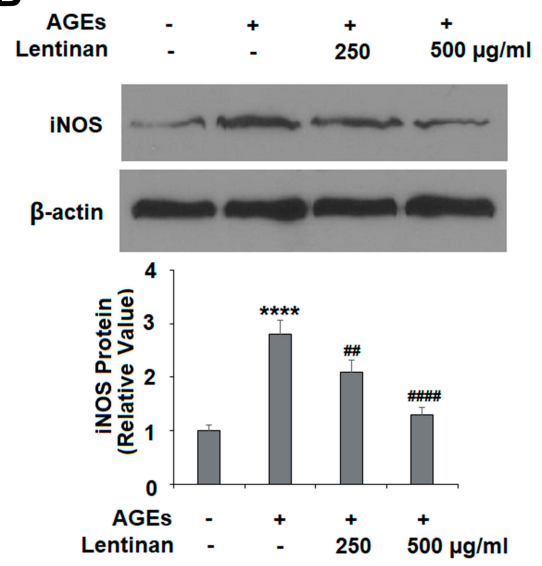

C
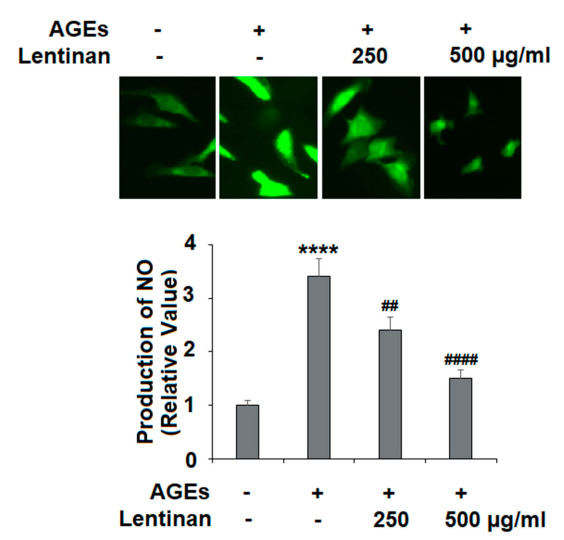

Figure 5 Lentinan inhibited AGE-induced iNOS expression and production of NO. Cells were stimulated with $100 \mu \mathrm{g} / \mathrm{mL}$ AGEs with or without lentinan (250 and $500 \mu \mathrm{g} /$ $\mathrm{mL}$ ) for 24 h. (A) mRNA of iNOS; (B) Protein of iNOS as measured by Western blot analysis; (C) Production of NO as measured by DAF-FM staining (****, P<0.000 I vs vehicle group; \#, \#, \#\#, P<0.05, 0.01, 0.0001 vs AGEs treatment group, n=4-5). 

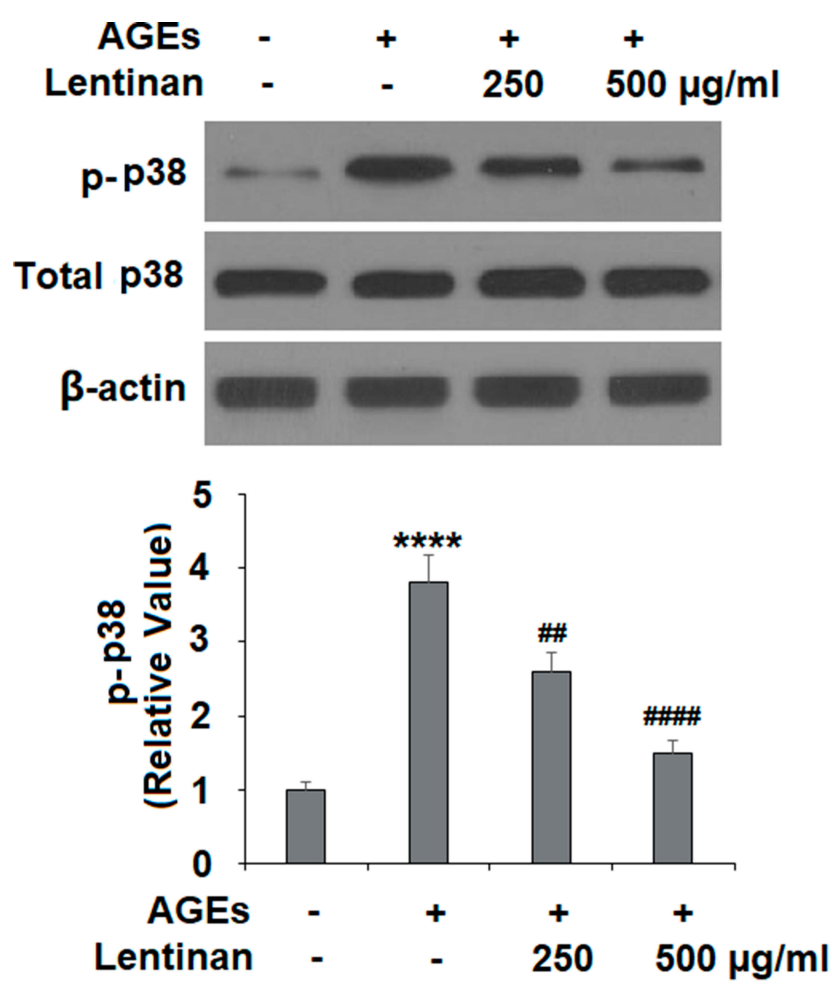

Figure 6 Lentinan inhibited AGE-induced activation of p38. Cells were stimulated with $100 \mu \mathrm{g} / \mathrm{mL}$ AGEs with or without lentinan $(250$ and $500 \mu \mathrm{g} / \mathrm{mL})$ for 2 h. Phosphorylated and total levels of $\mathrm{p} 38$ were measured $(* * * *, P<0.0001$ vs vehicle group; \#, \#, $\mathrm{P}<0.01,0.000 \mathrm{I}$ vs AGEs treatment group, $\mathrm{n}=4-5$ ).

NF- $\kappa \mathrm{B}$ was induced to 123.5 -fold by AGEs, which was ameliorated to 78.2- and 57.3-fold by lentinan in a dosedependent manner, respectively (Figure 8B).

\section{Discussion}

$\mathrm{OA}$ is a degenerative joint disease afflicting millions of people worldwide, primarily those over the age of $65 .{ }^{25}$ As cartilage degradation is a hallmark of the inflammatory process of OA, the inhibition of inflammatory factors that promote cartilage degradation has been shown to be an effective target for the treatment of OA. Although a large number of studies have proposed treatments for this disease, a safe and therapeutic strategy is still urgently needed. Lentinan, a polysaccharide derived from Lentinus edodes, has shown protective properties in inflammatory skin diseases, ${ }^{26}$ inflammatory bowel disease, ${ }^{27}$ and different cancers. ${ }^{28}$ In the present study, we employed lentinan to antagonize inflammation induced by AGEs in human chondrocytes. This is the first time the effect of lentinan in AGE-challenged chondrocytes has been measured. The results show that lentinan significantly suppresses the inflammatory response in chondrocytes.

When binding with RAGE, AGEs accumulate in the joints. The resulting excessive levels of AGEs stimulate chondrocytes to release various pro-inflammatory cytokines and chemokines such as IL- $1 \beta$, TNF- $\alpha$, and IL- 8 , which play a critical role in the progression of OA. ${ }^{29-31} \mathrm{IL}-1 \beta$ and TNF$\alpha$ have been demonstrated to upregulate the expression of ADAMTS and MMPs, especially MMP-1, MMP-3, and MMP-13, in chondrocytes. ${ }^{31,32}$ IL-8 plays a vital role in OA by attracting neutrophils and monocytes to promote an inflammatory response in chondrocytes. ${ }^{33,34}$ COX-2 and iNOS are two primary regulatory enzymes involved in various inflammatory responses that work by modulating the production of $\mathrm{PGE}_{2}$ and $\mathrm{NO}$, which are responsible for the development of inflammation. Furthermore, studies have reported that $\mathrm{PGE}_{2}$ and $\mathrm{NO}$ contribute to articular inflammation and destruction by increasing MMP activity and production. ${ }^{35,36}$ Lentinan, as a biological response modifier, has been proven to be safe and effective as a treatment and adjunctive therapy for inflammatory diseases. ${ }^{23,24}$ In our study, lentinan treatment significantly inhibited the expression of pro-inflammatory cytokines and chemokines, including IL- $1 \beta$, TNF- $\alpha$, IL-8, PGE $_{2}$, and NO. These results reveal that lentinan exerts anti-inflammatory effects in AGEinduced chondrocytes. In addition, as overexpression of proinflammatory cytokines and chemokines exists in various
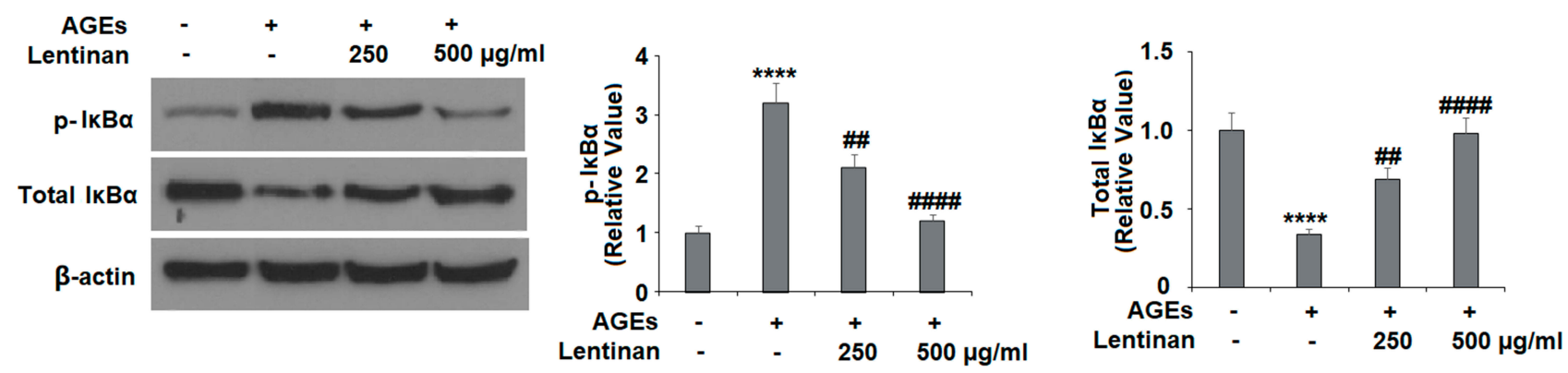

Figure 7 Lentinan inhibited AGE-induced phosphorylation and degradation of $\mathrm{I} \mathrm{B} \alpha$. Cells were stimulated with $100 \mu \mathrm{g} / \mathrm{mL}$ AGEs with or without lentinan ( 250 and $500 \mu g /$ $\mathrm{mL}$ ) for $2 \mathrm{~h}$. Phosphorylated and total levels of I $\mathrm{KB} \alpha$ were measured by Western blot analysis (****, $\mathrm{P}<0.000 \mathrm{I}$ vs vehicle group; \#, \#\#, P<0.0I, $0.000 \mathrm{I}$ vs $\mathrm{AGEs}$ treatment group, $n=4-5)$. 
A

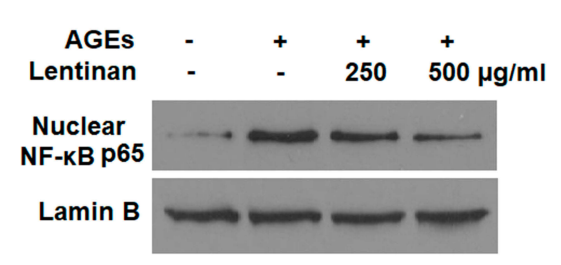

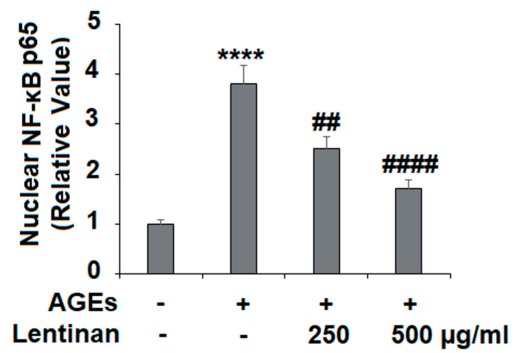

B

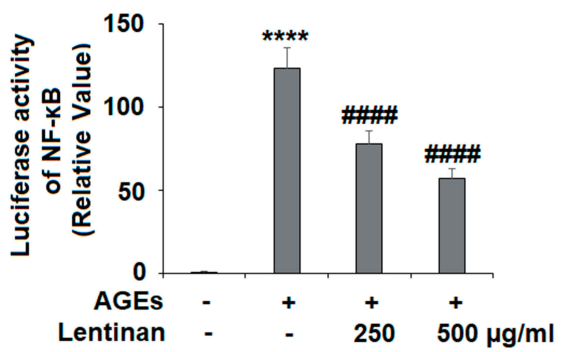

Figure 8 Lentinan inhibited AGE-induced activation of NF-kB. Cells were stimulated with $100 \mu g / \mathrm{mL}$ AGEs with or without lentinan (250 and $500 \mu g / \mathrm{mL})$ for 2 h. (A)

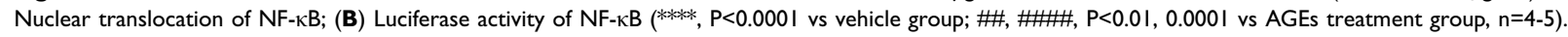

inflammatory diseases and promotes their progression and development, lentinan has the potential for applications in other related fields.

MMPs and ADAMTS are the primary hydrolytic proteinases involved in the destruction of the cartilage matrix in AGE-induced chondrocytes. ${ }^{37,38}$ Research has found that high expression of MMPs and ADAMTS, including MMP-1, MMP-3, MMP-13, ADAMTS-4, and ADAMTS-5, in chondrocytes induces the destruction of the cartilage matrix. On the other hand, the degraded products further promote the deterioration of the cartilage matrix, forming a vicious circle that accelerates cartilage matrix breakdown. ${ }^{39}$ Multiple studies have revealed that the production of MMPs and ADAMTS is modulated by the expression of pro-inflammatory cytokines and chemokines. ${ }^{35,36}$ In our study, AGE treatment significantly increased the production of MMPs and ADAMTS in chondrocytes. However, a disadvantage of this experiment is not being able to differentiate whether AGE treatment enhances the production of MMPs and ADAMTS directly or whether this occurs as an indirect result of the increase in the expression of pro-inflammatory cytokines. Regarding this, we speculate that lentinan might suppress the expression of MMPs and ADAMTS in a direct or indirect way. More research is required to demonstrate the inhibitory effect of lentinan on the expression of MMPs and ADAMTS. Our study suggests that lentinan has the potential to maintain the integrity of the cartilage matrix.

The NF- $\kappa \mathrm{B}$ signaling pathway plays a vital role in the progression and development of inflammatory diseases. In $\mathrm{OA}$, the NF- $\mathrm{BB}$ pathway has been proven to modulate the inflammatory response, production of cytokines, and degradation of the cartilage matrix. ${ }^{40}$ Under normal conditions, $\mathrm{NF}-\kappa \mathrm{B}$ is maintained in its inactive state in the cytoplasm by $\mathrm{I} \kappa \mathrm{B} \alpha$, a type of inhibitory protein. However, AGE stimulation leads to the activation of the p38/MAPK pathway and the phosphorylation of $\mathrm{I} \kappa \mathrm{B} \alpha$, resulting in nuclear translocation of p65 and the activation of NF- $\kappa \mathrm{B} .{ }^{41}$ When activated, NF- $\kappa \mathrm{B}$ upregulates the production of various of cytokines and chemokines such as IL- $1 \beta, \mathrm{TNF}-\alpha, \mathrm{PGE}_{2}$, and NO. These factors not only contribute to the development of the inflammatory response in OA chondrocytes, but also leads to the expression of MMPs and ADAMTS, which are responsible for the breakdown of the cartilage matrix. Our research provides evidence that lentinan inhibits the activation of the p38/MAPK pathway, thereby preventing I $\mathrm{B} \alpha$ from undergoing phosphorylation and degradation. Therefore, lentinan treatment modulates the activation of the NF- $\mathrm{BB}$ signaling pathway in a dose-dependent manner.

In this study, we reported that $250-500 \mu \mathrm{g} / \mathrm{mL}$ of lentinan displays a protective effect against AGE- induced inflammation and the expression of matrix-degrading enzymes in human SW1353 chondrocytes. Consistently, 250-500 $\mu \mathrm{g} / \mathrm{mL}$ lentinan has been shown to suppress inflammasome activation in bone marrow-derived macrophages. ${ }^{23}$ Additionally, $500 \mu \mathrm{g} /$ $\mathrm{mL}$ lentinan has displayed an anti-inflammatory capacity by inhibiting the expression of TNFR1 and IL-8 in Caco- 2 cells. ${ }^{24}$ Interestingly, several studies have used varied doses of chondroitin sulfate (CS) in cultured chondrocytes. For example, $10-25 \mu \mathrm{g} / \mathrm{mL} \mathrm{CS}$ is shown to be responsive for extracellular matrix metabolism. ${ }^{42,43}$ At high doses, $200 \mu \mathrm{g} / \mathrm{mL} \mathrm{CS}$ has been shown to suppress cytokine-induced chondrocyte activation. ${ }^{4,45}$ Based on these facts, we recognize that both lentinan and CS could be effective at doses of 100-200 $\mu \mathrm{g} / \mathrm{mL}$ in vitro. Importantly, previous studies have shown that CS treatment could promote the synthesis of $\mathrm{ECM}^{42}$ and exhibit anti-inflammatory effects in cultured chondrocytes. ${ }^{46,47} \mathrm{CS}$ also suppresses NF- $\kappa$ B accumulation in the nucleus. ${ }^{48} \mathrm{We}$ showed that lentinan suppresses AGE-induced expression of inflammatory cytokines, matrix metalloproteinases (MMPs), and the activation of NF- $\mathrm{KB}$ in chondrocytes. Therefore, we speculate that lentinan might have a comparable beneficial effect to CS. Based on the published studies on CS, it is 
typically administered orally at doses ranging from 800 to $1200 \mathrm{mg}$ /day. Previous work has shown that at 2 hour following oral administration, plasma levels of CS can reach 0.6-10 $\mu \mathrm{g} / \mathrm{mL}{ }^{49}$ However, other studies have administered lentinan via intraperitoneal injection. Compared to CS (molecular weight: $10-40 \mathrm{kDa}$ ), $500 \mathrm{kDa}$ lentinan is a much larger polysaccharide, which makes it difficult to pass through the semipermeable synovial membrane. Thus, the oral administration of lentinan could be long-term goal for investigation.

There are several limitations to the current study. Firstly, we investigated the effects of lentinan in human SW1353 chondrosarcoma cells but not in human primary chondrocytes. Investigation of primary chondrocytes is limited by a lack of a sufficient number of chondrocytes from operative procedures and the fact that after isolation, the biological and functional characteristics of primary chondrocytes differ considerably between donors and preparations [51]. Therefore, human SW1353 cells are widely used as a substitute for primary chondrocytes [52]. It should be noted that SW1353 cells exhibit only a limited chondrocytic phenotype in line with previous reports showing an epithelial phenotype of SW1353 cells after long-term culture [53]. Secondly, the pathological mechanisms of osteoarthritis are complicated. In addition to AGEs, there are a variety of toxins such as IL-1 $\beta$ that are associated with the progression and development of OA. Therefore, future in vivo studies using ideal animal models are necessary to clarify the beneficial effects of lentinan in $\mathrm{OA}$.

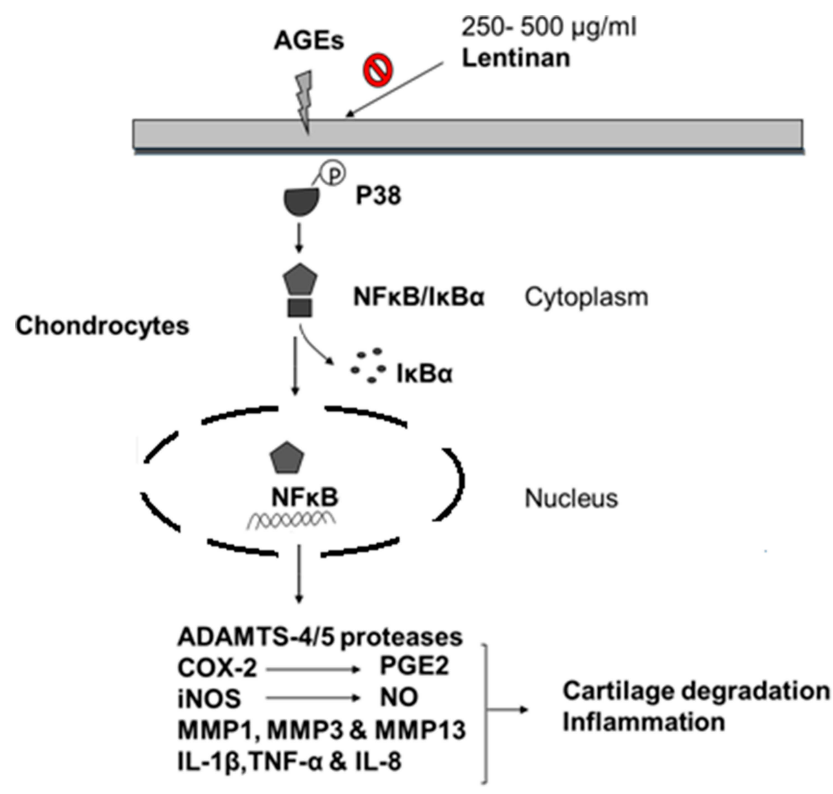

Figure 9 A representative schematic of the molecular mechanisms.
In conclusion, this study demonstrates the protective effects of lentinan in AGE-induced chondrocytes by inhibiting the production of pro-inflammatory cytokines and chemokines such as IL- $1 \beta$, TNF- $\alpha$, IL- 8, PGE $_{2}$, and $\mathrm{NO}$, suppressing the expression of regulatory enzymes including COX-2 and iNOS, and downregulating the expression of hydrolytic enzymes via the modulation of NF- $\kappa \mathrm{B}$ signaling by suppressing the phosphorylation of p38 and the phosphorylation and degradation of $\mathrm{I} \kappa \mathrm{B} \alpha$ (Figure 9). These findings suggest that lentinan may be effective in the treatment of chondrocyte-based inflammation in $\mathrm{OA}$.

\section{Ethics Statement}

The chondrosarcoma SW1353 cells used in all experiments were purchased from ATCC. All experiments were approved by the ethics committee of Henan Provincial Orthopedic Hospital.

\section{Disclosure}

The authors report no conflicts of interest in this work.

\section{References}

1. Rahmati M, Nalesso G, Mobasheri A, Mozafari M. Aging and osteoarthritis: central role of the extracellular matrix. Ageing Res Rev. 2017;40:20-30. doi:10.1016/j.arr.2017.07.004

2. Gelber AC, Hochberg MC, Mead LA, Wang NY, Wigley FM, Klag MJ. Joint injury in young adults and risk for subsequent knee and hip osteoarthritis. Ann Intern Med. 2000;133(5):321-328. doi:10.7326/0003-4819-133-5-200009050-00007

3. Berenbaum F, Eymard F, Houard X. Osteoarthritis, inflammation and obesity. Curr Opin Rheumatol. 2013;25(1):114-118. doi:10.1097/ BOR.0b013e32835a9414

4. Maillard LC. Action des acides amines sur les sucres: formation des melanoidines par voie methodique. Compt Rend. 1912;154:66-68.

5. Loeser RF, Yammani RR, Carlson CS, et al. Articular chondrocytes express the receptor for advanced glycation end products: potential role in osteoarthritis. Arthritis Rheum. 2005;52(8):2376-2385. doi:10.1002/ art.21199

6. Gao F, Zhang S. Salicin inhibits AGE-induced degradation of type II collagen and aggrecan in human SW1353 chondrocytes: therapeutic potential in osteoarthritis. Artif Cells Nanomed Biotechnol. 2019;47 (1):1043-1049. doi:10.1080/21691401.2019.1591427

7. Takahashi N, Harada M, Azhary JMK, et al. Accumulation of advanced glycation end products in follicles is associated with poor oocyte developmental competence. Mol Hum Reprod. 2019;25 (11):684-694. doi:10.1093/molehr/gaz050

8. Sun W, Gao Y, Ding Y, et al. Catalpol ameliorates advanced glycation end product-induced dysfunction of glomerular endothelial cells via regulating nitric oxide synthesis by inducible nitric oxide synthase and endothelial nitric oxide synthase. IUBMB Life. 2019;71(9):1268-1283. doi:10.1002/iub.2032

9. Matsumoto T, Kojima M, Takayanagi K, Katome T, Taguchi K. Amplification of the COX/TXS/TP receptor pathway enhances uridine diphosphate-induced contraction by advanced glycation end products in rat carotid arteries. Pflugers Arch. 2019;471(11-12):1505-1517. doi:10.1007/s00424-019-02330-y 
10. Nonaka K, Bando M, Sakamoto E, et al. 6-shogaol inhibits advanced glycation end-products-induced IL-6 and ICAM-1 expression by regulating oxidative responses in human gingival fibroblasts. Molecules. 2019;24(20):E3705. doi:10.3390/molecules24203705

11. Tong C, Liang H, Liu X, et al. The protective effects of exenatide against AGEs-induced articular matrix degradation in human primary chondrocytes. Am J Transl Res. 2019;11(4):2081-2089.

12. Stellavato A, Restaino OF, Vassallo V, et al. Comparative analyses of pharmaceuticals or food supplements containing chondroitin sulfate: are their bioactivities equivalent? Adv Ther. 2019;36(11):3221-3237. doi:10.1007/s12325-019-01064-8

13. Restaino OF, Finamore R, Stellavato A, et al. European chondroitin sulfate and glucosamine food supplements: a systematic quality and quantity assessment compared to pharmaceuticals. Carbohydr Polym. 2019;15(222):114984. doi:10.1016/j.carbpol.2019.114984

14. Restaino OF, Di Lauro I, Di Nuzzo R, De Rosa M, Schiraldi C. New insight into chondroitin and heparosan-like capsular polysaccharide synthesis by profiling of the nucleotide sugar precursors. Biosci Rep. 2017;37(1):1. doi:10.1042/BSR20160548

15. Stellavato A, Vassallo V, La Gatta A, et al. Novel hybrid gels made of high and low molecular weight hyaluronic acid induce proliferation and reduce inflammation in an osteoarthritis in vitro model based on human synoviocytes and chondrocytes. Biomed Res Int. 2019;23 (2019):4328219.

16. Stellavato A, De Novellis F, Reale S, De Rosa M, Schiraldi C. Hybrid complexes of high and low molecular weight: evaluation using an in vitro model of osteoarthritis. J Biol Regul Homeost Agents. 2016; Oct-Dec;30(4 Suppl 1):7-16.

17. Mizuno T. Development of anti-tumor polysaccharides from mushroom fungi, Food Foods Ingredients J. Jpn. 1996;167:69-85.

18. Ren G, Xu L, Lu T, Yin J. Structural characterization and anti-viral activity of lentinan from Lentinus edodes mycelia against infectious hematopoietic necrosis virus. Int $J$ Biol Macromol. 2018;115:1202-1210. doi:10.1016/j.ijbiomac.2018.04.132

19. Shirasaka T, Nakano K, Takechi T, et al. Anti-tumor effect of $1 \mathrm{M}$ tegafur-0.4 M 5-chloro-2,4- dihydroxypyridine-1 M potassium oxonate (S-1) against human colon carcinoma orthotopically implanted into nude rats. Cancer Res. 1996;56:2602-2606.

20. Ooi VEC, Liu F. Immunomodulation and anti-cancer activity of polysaccharide-protein complexes. Curr Med Chem. 2000;7:715-729. doi:10.2174/0929867003374705

21. Liu Y, Zhao J, Zhao Y, et al. Therapeutic effects of lentinan on inflammatory bowel disease and colitis-associated cancer. $J$ Cell Mol Med. 2019;23(2):750-760.

22. Liu Y, Zhao J, Zhao Y, et al. Therapeutic effects of lentinan on inflammatory bowel disease and colitis-associated cancer. $J$ Cell Mol Med. 2019;23(2):750-760.

23. Ahn H, Jeon E, Kim JC, et al. Lentinan from shiitake selectively attenuates AIM2 and non-canonical inflammasome activation while inducing pro-inflammatory cytokine production. Sci Rep. 2017;7 (1):1314. doi:10.1038/s41598-017-01462-4

24. Nishitani Y, Zhang L, Yoshida M, et al. Intestinal anti-inflammatory activity of lentinan: influence on IL-8 and TNFR1 expression in intestinal epithelial cells. PLoS One. 2013;8(4):e62441. doi:10.1371/journal.pone.0062441

25. Dillon CF, Rasch EK, Qiuping G, Hirsch R. Prevalence of knee osteoarthritis in the United States: arthritis data from the third national health and nutrition examination survey 1991-1994. J Rheumatol. 2006. 33(11):2271-2279.

26. Zi Y, Jiang B, He C, Liu L. Lentinan inhibits oxidative stress and inflammatory cytokine production induced by benzo(a)pyrene in human keratinocytes. J Cosmet Dermatol. 2019;28.

27. Liu Y, Zhao J, Zhao Y, et al. Therapeutic effects of lentinan on inflammatory bowel disease and colitis-associated cancer. $J$ Cell Mol Med. 2019;23(2):750-760.
28. Zhang M, Zhang Y, Zhang L, Tian Q. Mushroom polysaccharide lentinan for treating different types of cancers: A review of 12 years clinical studies in China. Prog Mol Biol Transl Sci. 2019;163:297-328.

29. Liu-Bryan R, Terkeltaub R. Emerging regulators of the inflammatory process in osteoarthritis. Nat Rev Rheumatol. 2015;11(1):35-44. doi:10.1038/nrrheum.2014.162

30. Kleine SA, Gogal RM, George C, Thaliath M, Budsberg SC. Elevated synovial fluid concentration of monocyte chemoattractant Protein-1 and Interleukin- 8 in dogs with osteoarthritis of the stifle. Vet Comp Orthop Traumatol. 2019;22.

31. Jia T, Qiao J, Guan D, Chen T. Anti-inflammatory effects of licochalcone A on IL-1 $\beta$-stimulated human osteoarthritis chondrocytes. Inflammation. 2017;40(6):1894-1902. doi:10.1007/s10753-017-0630-5

32. Tortorella MD, Malfait AM, Deccico C, Arner E. The role of ADAM-TS4 (aggrecanase-1) and ADAM-TS5 (aggrecanase-2) in a model of cartilage degradation. Osteoarthritis Cartilage. 2001;9:539-552. doi:10.1053/joca.2001.0427

33. Devitt BM, Queally JM, Vioreanu M, et al. Cobalt ions induce chemokine secretion in a variety of systemic cell lines. Acta Orthop. 2010;81(6):756-764. doi:10.3109/17453674.2010.537806

34. Lotz M, Terkeltaub R, Villiger PM. Cartilage and joint inflammation. Regulation of IL-8 expression by human articular chondrocytes. J Immunol. 1992;148:466-473.

35. Kapoor M, Martel-Pelletier J, Lajeunesse D, Pelletier JP, Fahmi H. Role of proinflammatory cytokines in the pathophysiology of osteoarthritis. Nat Rev Rheumatol. 2011;7(1):33-42. doi:10.1038/ nrrheum. 2010.196

36. Sun FF, Hu PF, Xiong Y, Bao JP, Qian J, Wu LD. Tricetin Protects rat chondrocytes against IL-1 $\beta$-induced inflammation and apoptosis. Oxid Med Cell Longev. 2019;2019:4695381. doi:10.1155/2019/ 4695381

37. Kobayashi M, et al. Role of interleukin-1 and tumor necrosis factor $\alpha$ in matrix degradation of human osteoarthritic cartilage. Arthritis Rheum. 2005;52:128-135. doi:10.1002/art.20776

38. Nagase H, Itoh Y, Binner S. Interaction of alpha 2-macroglobulin with matrix metalloproteinases and its use for identification of their active forms. Ann N Y Acad Sci. 1994;732:294-302. doi:10.1111/ j.1749-6632.1994.tb24744.x

39. Roman-Blas JA, Jimenez SA. NF- $\mathrm{kB}$ as a potential therapeutic target in osteoarthritis and rheumatoid arthritis. Osteoarthr Cartil. 2006;14 (9):839-848. doi:10.1016/j.joca.2006.04.008

40. Mohetaer M, Li G, Wang Y, et al. Protective effects of gemigliptin against type II collagen degradation in human chondrocytes. Biomed Pharmacother. 2018;104:590-594. doi:10.1016/j.biopha.2018.04.018

41. Mengshol JA, Vincenti MP, Coon CI, et al. Interleukin-1 induction of collagenase 3 (matrix metalloproteinase 13) gene expression in chondrocytes requires $\mathrm{p} 38$, c-jun $\mathrm{N}$-terminal kinase, and nuclear factor $\mathrm{jB}$ : differential regulation of collagenase 1 and collagenase 3 . Arthritis \& Rheumatol. 2000;43:801-811. doi:10.1002/1529-0131(200004) 43:4<801::AID-ANR10>3.0.CO;2-4

42. Wang L, Wang J, Almqvist KF, Veys EM, Verbruggen G. Influence of polysulphated polysaccharides and hydrocortisone on the extracellular matrix metabolism of human articular chondrocytes in vitro. Clin Exp Rheumatol. 2002;20(5):669-676.

43. Lippiello L. Osteoarthritis and Cartilage, Glucosamine and chondroitin sulfate: biological response modifiers of chondrocytes under simulated conditions of joint stress. 2003;11(5):335-342.

44. Calamia V, Ruiz-Romero C, Rocha B, et al. Pharmacoproteomic study of the effects of chondroitin and glucosamine sulfate on human articular chondrocytes. Arthritis Res Ther. 2010;12(4):R138. doi:10.1186/ar3077

45. Jomphe C, Gabriac M, Hale TM, et al. Chondroitin sulfate inhibits the nuclear translocation of nuclear factor-kappaB in interleukin-1beta-stimulated chondrocytes. Basic Clin Pharmacol Toxicol. 2008;102(1):59-65. 
46. Calamia V, Lourido L, Fernández-Puente P, et al. Secretome analysis of chondroitin sulfate-treated chondrocytes reveals anti-angiogenic, anti-inflammatory and anti-catabolic properties. Arthritis Res Ther. 2012;14(5):R202. doi:10.1186/ar4040

47. Stellavato A, Tirino V, de Novellis F, et al. Biotechnological chondroitin a novel glycosamminoglycan with remarkable biological function on human primary chondrocytes. $J$ Cell Biochem. 2016;117(9):2158-2169. doi:10.1002/jcb.25556
48. Jomphe CJ, Gabriac M, Hale TM, et al. Chondroitin sulfate inhibits the nuclear translocation of nuclear factor-kappaB in interleukin-lbeta-stimulated chondrocytes. Basic Clin Pharmacol Toxicol. 2008;102:59-65. doi:10.1111/j.1742-7843.2007.00158.x

49. Volpi N. Oral bioavailability of chondroitin sulfate (Condrosulf) and its constituents in healthy male volunteers. Osteoarthritis Cartilage. 2002;10:768-777. doi:10.1053/joca.2002.0824

\section{Publish your work in this journal}

Drug Design, Development and Therapy is an international, peerreviewed open-access journal that spans the spectrum of drug design and development through to clinical applications. Clinical outcomes, patient safety, and programs for the development and effective, safe, and sustained use of medicines are a feature of the journal, which has also been accepted for indexing on PubMed Central. The manuscript management system is completely online and includes a very quick and fair peer-review system, which is all easy to use. Visit http://www. dovepress.com/testimonials.php to read real quotes from published authors. 